\title{
Capacitação sobre vacina para os agentes comunitários de saúde: relato de experiência de residentes em saúde coletiva
}

Training on vaccine for community health agents: report on the experience of residents in collective health

Formación en vacuna para agentes de salud comunitarios: informe sobre la experiencia de residentes en salud colectiva

Recebido: 28/08/2021 | Revisado: 02/09/2021 | Aceito: 09/09/2021 | Publicado: 12/09/2021

\author{
Diana Loyce Monteiro \\ ORCID: https://orcid.org/0000-0002-3938-6911 \\ Escola de Governo em Saúde Pública de Pernambuco, Brasil \\ E-mail: dianaloyce@gmail.com \\ Ana Carolina dos Santos Anjos \\ ORCID: https://orcid.org/0000-0003-1475-3831 \\ Escola de Governo em Saúde Pública de Pernambuco, Brasil \\ E-mail: carolsantostj@hotmail.com \\ Rafaela de Oliveira Xavier \\ ORCID: https://orcid.org/0000-0002-2122-6418 \\ Escola de Governo em Saúde Pública de Pernambuco, Brasil \\ E-mail: rafaela_ox09@hotmail.com \\ Renata de Oliveira Cartaxo \\ ORCID: https://orcid.org/0000-0002-6720-0897 \\ Universidade de Pernambuco, Brasil \\ E-mail: renacartaxo@gmail.com
}

\begin{abstract}
Resumo
Introdução: $\mathrm{O}$ processo de territorialização possibilita o conhecimento acerca das necessidades da população subsidiando o planejamento de ações em saúde. Nesse contexto, o Agente Comunitário de Saúde faz o principal elo entre a comunidade e a Unidade Básica de Saúde, e dentre suas atribuições está o acompanhamento da situação vacinal. Dessa forma, o aperfeiçoamento profissional nessa temática é de fundamental importância para sua correta divulgação no território. Objetivo: Descrever a experiência das residentes em Saúde Coletiva sobre atividade desenvolvida em uma Unidade Básica de Saúde. Métodos: Trata-se de um relato de experiência resultante de uma atividade de capacitação sobre calendário vacinal, desenvolvida para os agentes comunitários de saúde de uma Unidade Básica do município de Salgueiro-PE. Resultados: O momento foi ministrado pela coordenadora regional do PNI que iniciou realizando questionamentos sobre a temática possibilitando a troca de experiências entre os participantes. Em seguida, a palestrante explanou sobre o calendário vacinal e as principais campanhas de vacinação. Ao final foi entregue um material impresso produzido pelas residentes contendo as vacinas distribuídas por faixa etária, a fim de se tornar um instrumento de trabalho para os agentes. Considerações Finais: O presente trabalho ressalta a importância da constante capacitação para que os profissionais de saúde consigam repassar à população informações atualizadas e corretas. Além disso, contribui para o processo de formação das residentes em Saúde Coletiva de forma que consigam entender o papel da atenção básica dentro da Rede de Atenção à Saúde.
\end{abstract}

Palavras-chave: Agentes comunitários de saúde; Capacitação profissional; Saúde coletiva.

\begin{abstract}
Introduction: The territorialization process enables knowledge about the population's needs, supporting the planning of health actions. In this context, the Community Health Agent is the main link between the community and the Basic Health Unit, and among their attributions is the monitoring of the vaccination situation. Thus, professional improvement in this theme is of fundamental importance for its correct dissemination in the territory. Objective: To describe the experience of residents in Public Health about activities developed in Primary Care. Methods: This is an experience report resulting from a training activity on the vaccination calendar, developed for community health workers in a Basic Unit in the municipality of Salgueiro-PE. Results: The moment was given by the regional coordinator of the PNI, who started by asking questions about the theme, enabling the exchange of experiences among the participants. Then, the speaker explained about the vaccination calendar and the main vaccination campaigns. At the end, a printed material produced by the residents was delivered, containing the vaccines distributed by age group, in order to become a working tool for the agents. Final Considerations: This study highlights the importance of
\end{abstract}


constant training so that health professionals can provide the population with up-to-date and correct information. In addition, it contributes to the process of training residents in Public Health so that they can understand the role of primary care within the Health Care Network.

Keywords: Community health agents; Professional training; Public health.

\section{Resumen}

Introducción: El proceso de territorialización permite conocer las necesidades de la población, apoyando la planificación de acciones de salud. En este contexto, el Agente Comunitario de Salud es el vínculo principal entre la comunidad y la Unidad Básica de Salud, y entre sus atribuciones está el seguimiento de la situación de vacunación. Por tanto, la superación profesional en esta temática es de fundamental importancia para su correcta difusión en el territorio. Objetivo: Describir la experiencia de los residentes de Salud Pública sobre las actividades desarrolladas en Atención Primaria. Métodos: Se trata de un relato de experiencia resultante de una actividad de capacitación sobre el calendario de vacunación, desarrollada para trabajadores comunitarios de salud en una Unidad Básica del municipio de Salgueiro-PE. Resultados: El momento estuvo a cargo de la coordinadora regional del PNI, quien comenzó haciendo preguntas sobre el tema, posibilitando el intercambio de experiencias entre los participantes. A continuación, el ponente explicó sobre el calendario de vacunación y las principales campañas de vacunación. Al finalizar, se entregó un material impreso elaborado por los vecinos, que contiene las vacunas distribuidas por grupos de edad, con el fin de que se convierta en una herramienta de trabajo para los agentes. Consideraciones finales: Este trabajo destaca la importancia de la capacitación constante para que los profesionales de la salud puedan brindar a la población información actualizada y correcta. Además, contribuye al proceso de formación de los residentes en Salud Pública para que comprendan el papel de la atención primaria dentro de la Red Asistencial.

Palabras clave: Agentes comunitarios de salud; Capacitación profesional; Salud pública.

\section{Introdução}

A descentralização do Sistema Único de Saúde (SUS) passou por transformações nas suas diretrizes onde foi possível observar o direcionamento para a promoção de saúde da população ao invés do foco apenas no tratamento de doenças, neste sentido, é necessário estabelecer formas contínuas de acompanhamento e cuidado. Para que isto aconteça de forma eficaz, os profissionais de saúde precisam criar vínculos na área pertencente à abrangência da Unidade Básica de Saúde (UBS) (Losco \& Gemma, 2019).

Para se conhecer bem o território é fundamental que haja compreensão das necessidades e problemas de saúde da população, tendo em vista o estabelecimento de vínculos entre os serviços de saúde e a comunidade e a construção de relações através das situações econômicas, sociais e situação de saúde. A investigação do campo do território é capaz de reconhecer os fatores de determinação social presente nos processos saúde-doença e reorientar o modelo de atenção (Gomes, Pinto, Cassuce, 2021).

Esse processo é realizado por meio da territorialização, que se apresenta como ferramenta fundamental para o planejamento de ações em saúde, possibilitando o desenvolvimento de intervenções epidemiológicas e atividades voltadas às problemáticas identificadas. A territorialização constitui-se como um elo entre o profissional de saúde e a comunidade, trazendo a Atenção Primária à Saúde como eixo central das políticas de saúde pública, pois sendo ela uma porta de entrada do SUS, cada membro da equipe deve conhecer e desenvolver seu papel na área de abrangência da UBS (Araújo, Alves Filho, Santos, Lira, 2017).

Dentre os profissionais que atuam na Atenção Primária, destacam-se os Agentes Comunitários de Saúde (ACS), trabalhadores com uma identidade comunitária e residentes da área de atuação, sendo o principal mediador entre a comunidade e os profissionais da UBS (Simas \& Pinto, 2017). O trabalho do ACS é complexo e inclui ações de promoção e vigilância à saúde, como pré-natal, imunização, acompanhamento de doenças crônicas não transmissíveis, cuidado com idosos, entre outros (Barreto et al., 2018).

Nesse sentido, é importante a valorização e reconhecimento do ACS como peça fundamental no sistema de saúde, sendo que a realização de capacitações de forma efetiva desses atores é uma forma de contribuir para essa valorização, uma vez que essas são insuficientes, e não há um preparo devido desses profissionais para atuarem diante os problemas que se 
deparam no seu exercício de trabalho. É notório a necessidade de maiores investimentos, pois é através da educação permanente, que os ACS poderão proporcionar maior eficiência nas funções de promoção da saúde e prevenção de agravos em sua comunidade (Almeida et al., 2021).

A erradicação de inúmeras morbidades no Brasil, foi possível devido ao intenso trabalho de imunização disseminada em crianças da primeira infância e o esforço dos profissionais que atuam no SUS, dentre eles o ACS. A imunização compreende como um dos cuidados primários de saúde e proporciona resposta quase que imediata na prevenção de doenças, sendo importante que o indivíduo receba todas as doses necessárias nas datas corretas, para que se tenha qualidade e eficácia no processo de imunização (Almeida et al., 2021)

Sendo assim, entre os assuntos relevantes para serem abordados na educação permanente, a vacinação é primordial pois faz parte da rotina dos profissionais da UBS que verifica a caderneta, situação vacinal e encaminha a população à Unidade para iniciar ou completar o esquema vacinal, conforme os calendários de vacinação (Brasil, 2014).

Nesse último aspecto citado, o ACS está entre os profissionais que contribuem para a divulgação das campanhas vacinais na comunidade (Brasil, 2009). Sendo assim, é fundamental que haja aperfeiçoamento sobre o assunto, pois uma vez que eles estão com base científica e atualizada, podem contrapor a circulação de notícias falsas sobre a importância e os benefícios das vacinas.

Os residentes multiprofissionais em saúde coletiva quando inseridos nos territórios de exposição, tem o objetivo de responder às necessidades de saúde com ações e intervenções que estimulem a construção de conhecimento (Ramos, 2006). Portanto, o presente trabalho visa relatar a experiência das atividades desenvolvidas por esses profissionais quando inseridos na atenção básica. Use o parágrafo como modelo.

\section{Metodologia}

Trata-se de um relato de experiência de duas residentes, do Programa de Residência Multiprofissional em Saúde Coletiva com Ênfase em Gestão de Redes de Saúde da Escola de Governo em Saúde Pública de Pernambuco - ESPPE, fruto de ações vivenciadas na Unidade Básica de Saúde (UBS) Rita Alves dos Santos ou UBS Cohab, localizada no município de Salgueiro-PE, no período de outubro de 2020. Utilizou-se a técnica de observação direta participante, uma vez que as duas observadoras do fenômeno também compunham o momento observado. A anotação em diário de campo entre as duas pesquisadoras deu-se individualmente com posterior reunião das observações anotadas à luz dos objetivos constituídos para este momento de formação.

As residentes em saúde coletiva formam suas experiências teórico-práticas através de ciclos de rodízios em coordenações e gerências de saúde em âmbitos municipais e estaduais presentes no município de Salgueiro-PE, tendo como apoio ou referência principal a VII Gerência Regional de Saúde de Pernambuco (GERES).

Na UBS descrita, as residentes contam com a preceptoria da profissional de enfermagem presente no local ao qual orienta e acolhe os profissionais em formação nas suas devidas atividades. A preceptora tem como função acompanhar a participação das residentes nos serviços, subsidiando produtos que podem ser elaborados para benefício do local ou dos trabalhadores de saúde da UBS.

Neste sentido, para identificar o tema da capacitação para os ACS, foram realizadas reuniões com a preceptora junto com os ACS que compõem a UBS. Durante as conversas, percebeu-se a necessidade de abordar sobre o calendário vacinal visto ser um tema recorrente de dúvidas da população e a confeccionar um material de apoio para acompanhar o ACS nas visitas domiciliares.

A partir destas observações, as residentes em saúde coletiva buscaram reunir-se com as coordenadoras regionais da pasta de Atenção Básica e Programa Nacional de Imunização (PNI), locadas na VII GERES, para organizar o evento. No 
decorrer do encontro, foi construído um esboço contendo o passo a passo da capacitação que abordaria: acolhimento da equipe, atividades de problematização com o objetivo de reconhecer o conhecimento deles sobre o assunto, slides dinâmicos a fim de promover o debate para trocas de experiências, vídeos autoexplicativos sobre o tema e dinâmica final.

Para melhor partilha da ideia, ainda foram convidados representantes da Secretaria Municipal de Saúde de SalgueiroPE, que foi referenciado pelo coordenador que continha mais acesso aos ACS da UBS, a saber, o colaborador responsável pelo Programa Bolsa Família. Ele também participou na construção da proposta de ação.

Para atrair o público-alvo para a capacitação, ou seja, os Agentes Comunitários de Saúde, elaborou-se um convite contendo informações, como: data, local, palestrante e as ofertas que aconteceriam no dia do evento. As residentes fizeram a circulação em meio digital e reforçaram diariamente sobre a ação com a equipe de saúde.

A fim de evitar propagação do COVID-19, as residentes traçaram meios de medidas preventivas para o dia capacitação, como: distanciamento das cadeiras na sala de reuniões, disposição de álcool $70^{\circ}$ e máscaras descartáveis. Além disso, no texto do convite havia recomendação de isolamento social para os profissionais que estivessem com sintomas gripais e reposição com material teórico para eles.

\section{Resultados}

O evento ocorreu pela manhã, na sala de reuniões da VII Gerência Regional de Saúde, onde obteve-se a participação de dez Agentes Comunitários de Saúde lotados na UBS Cohab, além dos atores que apoiaram a construção da capacitação, como: coordenadora regional de atenção básica, coordenadora regional do Programa Nacional de Imunização (PNI), coordenador municipal do Programa Bolsa Família, enfermeira da Unidade Básica de Saúde e técnicas de enfermagem. Na Figura 1, é possível visualizar o material individual personalizado, de proteção e de curso, disponibilizado para os participantes da formação.

Figura 1: Material de apoio.

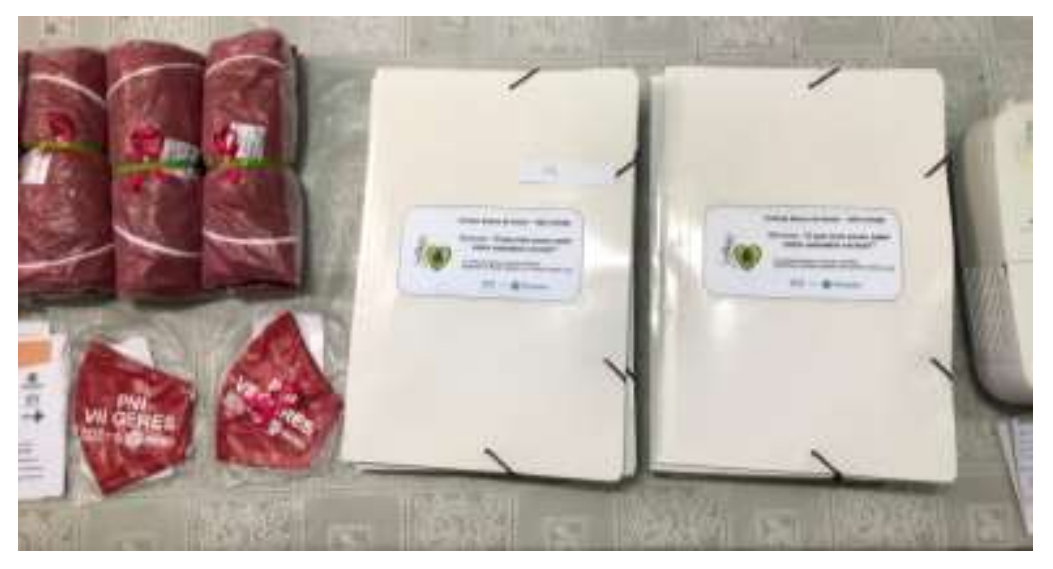

Fonte: fotografia autoral.

No acolhimento de boas-vindas, foi confeccionada uma caixinha contendo mensagens aleatórias sobre motivação, equilíbrio e empatia no ambiente de trabalho. Uma das residentes fez uma reflexão tendo como base o poema "Amor" do autor Carlos Drummond de Andrade, logo após, convidou cada participante a retirar uma mensagem da caixinha. A maioria dos presentes relatou se identificar com a mensagem sorteada e sinalizou que estava precisando ouvir aquelas palavras naquela manhã.

Este momento foi fundamental para que os participantes pudessem enxergar o valor pessoal e individual de cada um 
perante a equipe. Devido às restrições do COVID-19, alguns ACS precisaram se afastar do seu exercício de profissão e verbalizaram a saudade de sentir-se úteis no período pandêmico. Por meio do acolhimento, foi possível ouvir os sentimentos dos presentes e sua expectativa para aquela capacitação.

$\mathrm{Na}$ atividade inicial, a palestrante fez questionamentos inquietantes sobre calendário vacinal, propondo que os participantes se dividissem em trios e respondessem quais eram as vacinas recomendadas para determinada faixa etária. Foi orientado que não pesquisassem as respostas na internet, mas finalizasse o resultado de acordo com seus conhecimentos. Cada trio recebeu uma cartolina com hidrocores coloridos para realizar o esquema das respostas. Após a finalização, houve discussão do assunto com o intuito de sanar qualquer dúvida existente.

Essa dinâmica possibilitou que a palestrante identificasse o nível de conhecimento dos Agentes Comunitários sobre o assunto, oportunizando que ela pudesse dialogar de acordo com a necessidade do grupo. Além disso, promoveu o primeiro momento de troca de experiências entre palestrante, ACS e as residentes, permitindo que os presentes conhecessem a realidade do trabalho um dos outros e identificassem novas formas de resolução de problemas.

A palestra durou cerca de 50 minutos e teve embasamento teórico no Manual de Normas e Procedimentos para Vacinação (2020) fornecido pelo Ministério de Saúde e outras literaturas viabilizadas pelo estado de Pernambuco. Na oportunidade, foi reforçado quais eram as campanhas de vacina vigentes naquele mês e qual seria a melhor forma de divulgação devido às circunstâncias de restrição do COVID-19. O grupo concluiu a estratégia de comunicação através das mídias digitais ofereceriam suporte necessário para a propagação da informação somadas à atuação das rádios comunitárias presente na rotina da comunidade.

Durante a capacitação, houve a passagem de um vídeo sobre "A revolta da vacina" para fomentar a comunicação dialógica, trazendo um olhar para o passado com a reflexão do presente. Apesar da maioria dos ACS recomendarem vacinas diariamente, foi possível notar que poucos sabem as conquistas e desafios da aplicação do calendário vacinal no Brasil bem como sobre os mitos e verdades desse assunto.

$\mathrm{O}$ material de apoio que foi desenvolvido pelas residentes consistiu em um folder didático contendo o calendário vacinal referente a cada faixa etária. As informações contidas no instrumento foram retiradas do site do Ministério da Saúde e confirmadas com a coordenadora estadual do Programa Nacional de Imunização, como visualizamos na Figura 2. Todas as cópias foram impressas em colorido e plastificadas, a fim de que os ACS pudessem carregar com facilidade dentro das suas pastas nas visitas domiciliares.

De acordo com a maioria dos ACS, quando eram questionados sobre vacina e não sabiam devidamente a resposta, eles entravam em contato com a equipe da UBS ou retornavam a mesma e só em outro momento, conseguiam responder ao sujeito. Portanto, o folder tinha como objetivo possibilitar o repasse de informações de forma rápida e segura para a comunidade. 
Figura 2: Material educativo impresso.
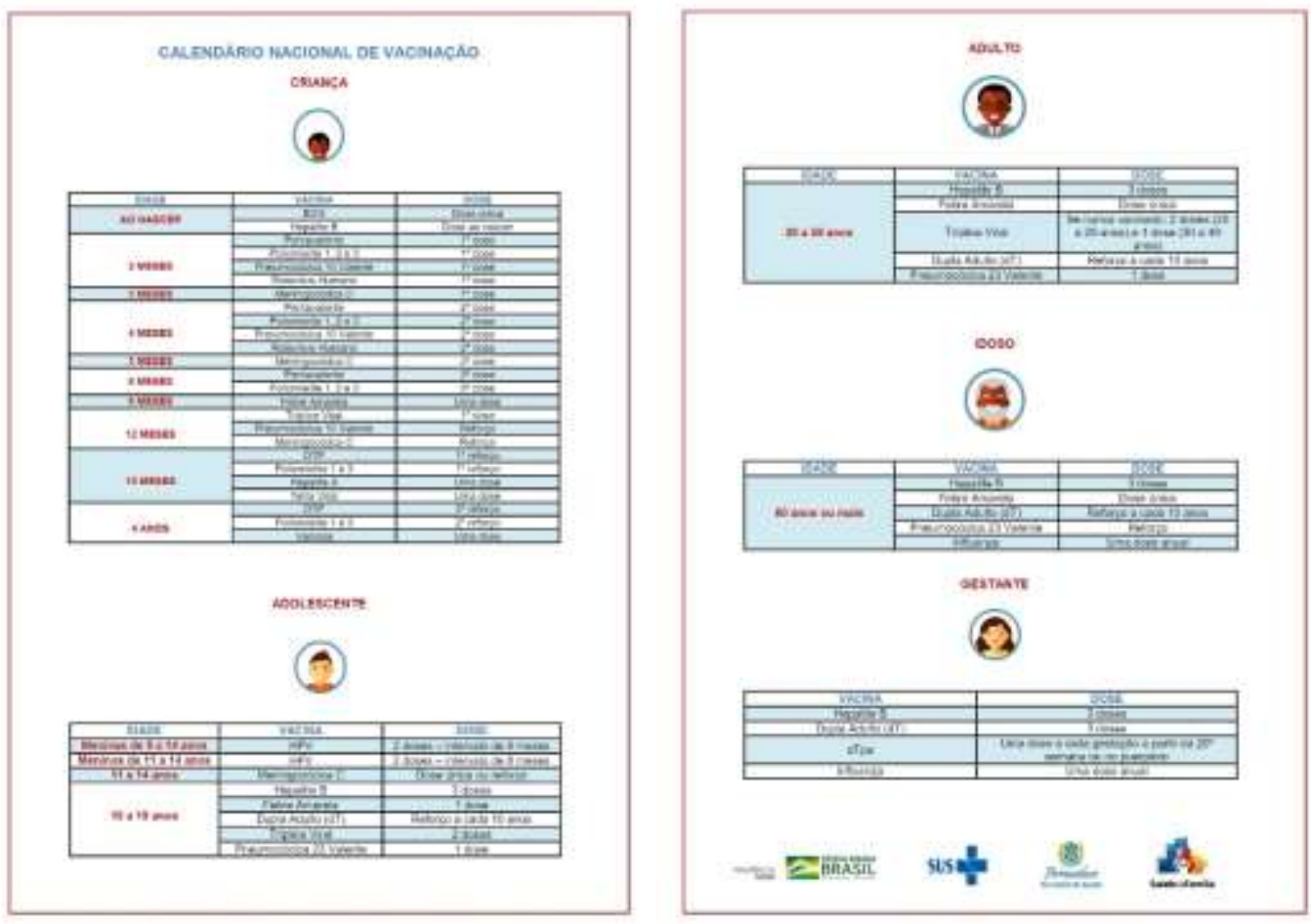

Fonte: Elaboração própria.

No final do encontro, os ACS foram convidados a participar de uma dinâmica completando a seguinte frase "ACS de verdade é aquele que...”. Neste momento, o público-alvo conseguiu expor que o verdadeiro profissional tem amor pela comunidade ao qual trabalha e é pertencente. Muitos também verbalizaram que o autêntico ACS tem disposição para encarar os desafios do seu exercício.

Logo depois, todos os presentes puderam receber um certificado com carga horária total para registrar o momento final da capacitação, como pode-se notar na Figura 3. Os ACS participaram do sorteio de brindes disponibilizado em caráter pessoal pela coordenadora regional do Programa Nacional de Imunização, como máscaras personalizadas e camisas.

Figura 3: Entrega do material para os participantes.

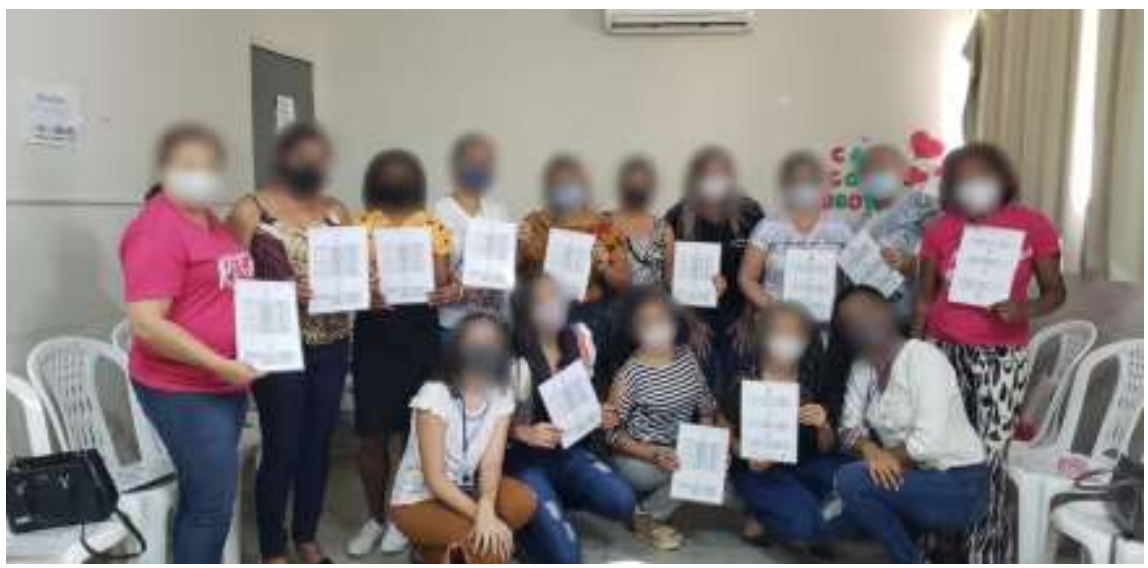

Fonte: Fotografia autoral. 
Esse encerramento permitiu indiretamente que cada ACS autoavaliasse seu trabalho, podendo reconhecer dentro do grupo alguma característica sua ou de necessidade do serviço. De forma geral, notou-se que os ACS reconhecem as dificuldades, mas estão dispostos a encará-las pois tem como foco atender a comunidade que se configuram em seus amigos e familiares.

\section{Discussão}

A Educação Permanente em Saúde (EPS) é um incentivo para aumentar os processos de ensino e aprendizagem para os profissionais de saúde, contribuindo para o melhor desempenho de suas funções. Nesse contexto, executar a EPS é de extrema importância para se ofertar serviços de qualidade, pois relaciona o conhecimento teórico com as vivências e experiências profissionais do dia a dia (Almeida et al., 2016).

$\mathrm{Na}$ Atenção Básica, os agentes comunitários de saúde assumem um papel fundamental devido ao seu amplo conhecimento sobre o território, o que permite o fortalecimento do elo entre usuários e equipe de saúde (Guanaes-Lorenzi, 2016). Segundo Zerbeto, Carvalho, Rossa e Paula (2020), manter o ACS capacitado favorece a identificação mais assertiva das necessidades do território, reduzindo os fatores de risco ali presentes e contribuindo para a melhora da qualidade de vida.

Uma das atribuições do ACS é realizar o acompanhamento da caderneta de vacinação dos moradores de sua microárea. Sabe-se que manter a vacinação em dias aumenta a prevenção das doenças sendo um método extremamente eficaz na saúde pública (Araújo, Sousa \& Pinho, 2019). Martins et al. (2019) evidenciam em seu estudo a necessidade de ampliar as ações de capacitação em vacinas para toda a equipe da $\mathrm{AB}$, principalmente os ACSs, já que eles são responsáveis pela busca ativa nos territórios.

Dentre as diversas maneiras de se organizar o momento de capacitação profissional, as residentes optaram pela utilização de dinâmicas de grupo, divididas em três momentos: acolhimento, discussão da temática e encerramento. Tal escolha corrobora com os achados de Massaroli (2016), que salientou os benefícios dessa ação, com destaque para a participação ativa dos convidados e o aumento das suas competências no assunto abordado.

Outro aspecto explorado na capacitação aqui exposta foi a utilização da metodologia da problematização antecedendo a fala da palestrante. Esse método consiste na exploração da troca de conhecimentos e vivências sobre o tema em questão com o objetivo de fortalecer o pensamento crítico-reflexivo de acordo com a realidade local (Ribeiro et al., 2016). Dessa forma, a discussão sobre o calendário vacinal se tornou mais focada nas principais dúvidas apresentadas e no conhecimento prévio sobre a temática.

A utilização de materiais educativos impressos (MEI), tais como cartilhas, panfletos e folders, é uma ótima ferramenta para complementar o processo de educação em saúde (Massara et al., 2016). Na atividade detalhada no presente trabalho, foi utilizada uma forma de comunicação em saúde através da confecção de material de apoio, cujo objetivo foi de auxiliar os agentes comunitários durante suas atividades diárias, transformando o folder em instrumento de trabalho.

De acordo com Sanchez, Lemos e Veríssimo (2017), o material escrito é uma das opções de MEI que possibilita o aumento do conhecimento através da leitura, ampliando a oferta de informações e servindo como apoio em caso de dúvidas tanto pelos profissionais de saúde como pelos usuários do SUS. Esse foi o principal propósito das residentes durante a capacitação dos agentes comunitários de saúde.

Sendo assim, a atividade desenvolvida contribuiu para a formação profissional das residentes em saúde coletiva, além de ajudar a equipe da UBS a identificar as fragilidades do serviço e traçar estratégias para resolvê-las (Domingos, 2015). A presença das residentes também fortaleceu o vínculo entre a equipe, proporcionando a melhoria do cuidado e atenção aos usuários, assim como mostra o estudo de Oliveira e Medeiros (2018). 


\section{Considerações Finais}

O trabalho em questão foi relevante para o processo de formação das residentes em saúde coletiva, pois permitiu que elas reconhecessem o valor da atenção básica na Rede de Atenção à Saúde (RAS) e no Sistema Único de Saúde (SUS). O contato presencial com os usuários ampliou o olhar para a gestão em saúde dentro da sua integralidade.

A partir da construção do evento foi possível compreender a importância do planejamento multiprofissional dentro da Unidade Básica de Saúde e subsidiou possibilidades de conhecer melhor o território bem como as necessidades de formação dos Agentes Comunitários de Saúde (ACS), aproximando o vínculo entre esses atores.

A abordagem sobre calendário vacinal com os ACS demonstrou que os mesmos têm aptidão para aprender novos conhecimentos, denotando interesse em partilhar o que já sabem a respeito do assunto e em repassar os novos aprendizados para a comunidade. Por consequência, a população é beneficiada com informações atuais e corretas.

Neste trabalho, notou-se que a educação permanente é essencial para que os profissionais se sintam atualizados e capacitados para exercerem sua função de forma segura e responsável e como a presença da Residência Multiprofissional em Saúde Coletiva com Ênfase em Gestão de Redes de Saúde qualifica essa experiência. A APS é um local com grande potencial para o desenvolvimento dessa prática fortalecendo o trabalho em equipe e o vínculo com os usuários, e reforçando o modelo de atenção â saúde vigente e, embora a EPS seja uma atividade de caráter rotineiro, proporcionar momentos específicos de formação, com ambiência pensada, metodologias horizontais e certificação valorizam o momento de educação para o trabalho daquele grupo. A integração entre a APS e a atuação da residência possibilita a qualificação dos espaços formadores e subsidia uma prática do trabalho vivo em saúde baseado na palavra-reflexão-ação e deve ser sempre estudada em seus processos para melhoramento de seus resultados.

\section{Agradecimentos}

Agradecemos à toda equipe da Unidade Básica de Saúde (UBS) Rita Alves dos Santos ou UBS Cohab, do município de Salgueiro-PE.

\section{Referências}

Almeida, A. O. S., Oliveira, A. M. B., Martins, A. C. G. S., Costa, N. P., Martins, T. S., Costa, N. P., Martins, T. S., Pereira, N. M. L., Almeida, B. R. R., Sousa, M. N. L., Silva, R. L., Noronha, R. C. R., Tavares, F. S., Frade, L. F. S., Lobão, G. C. A., \& Lago, S. J. C. (2021). O conhecimento dos Agentes Comunitários de Saúde sobre o calendário vacinal infantil. Research, Society and Development, 10(7). http://dx.doi.org/10.33448/rsd-v10i7.16591.

Almeida, J. R. de S., Bizerril, D. O., Saldanha, K. G. de H., \& Almeida, M. E. L. de. (2016). Educação Permanente em Saúde: uma estratégia para refletir sobre o processo de trabalho. Revista Da ABENO, 16(2), 7-15.

Araújo, G. B., Filho, F. W. P. A., Santos, R. da S., \& Lira, R. C. M. (2017). Territorialização em saúde como instrumento de formação para estudantes de medicina: relato de experiência. SANARE - Revista de Políticas Públicas, 16(1), 124-129.

Araújo, T. M. de, Souza, F. de O., \& Pinho, P. de S. (2019). Vacinação e fatores associados entre trabalhadores da saúde. Cadernos de Saúde Pública, 35(4).

Barreto, I. C. de H. C., Pessoa, V. M., Sousa, M. de F. A. de, Nuto, S. de A. S., Freitas, R. W. J. F. de, Ribeiro, K. G., Vieira-Meyer, A. P. G. F., \& Andrade, L. O. M. de. (2018). Complexidade e potencialidade do trabalho dos Agentes Comunitários de Saúde no Brasil contemporâneo. Saúde Em Debate, 42(spe1), $114-129$.

Barros, L. S. de, \& Cecílio, L. C. de O. (2019). Entre a "grande política" e os autogovernos dos Agentes Comunitários de Saúde: desafios da micropolítica da atenção básica. Saúde Em Debate, 43(spe6), 10-21.

Brasil. (2009). Ministério da Saúde. Secretaria de Atenção à Saúde. Departamento de Atenção Básica. O trabalho do agente comunitário de saúde. Ministério da Saúde - Brasília, 2009.

Brasil. (2014). Ministério da Saúde. Secretaria de Vigilância em Saúde. Departamento de Vigilância das Doenças Transmissíveis. Manual de Normas e Procedimentos para Vacinação. Ministério da Saúde - Brasília, 2014.

Domingos, C. M., Nunes, E. de F. P. de A., \& Carvalho, B. G. (2015). Potencialidades da Residência Multiprofissional em Saúde da Família: o olhar do trabalhador de saúde. Interface - Comunicação, Saúde, Educação, 19(55), 1221-1232. 
Research, Society and Development, v. 10, n. 12, e23101219963, 2021

(CC BY 4.0) | ISSN 2525-3409 | DOI: http://dx.doi.org/10.33448/rsd-v10i12.19963

Guanaes-Lorenzi, C., \& Pinheiro, R. L. (2016). A (des)valorização do agente comunitário de saúde na Estratégia Saúde da Família. Ciência \& Saúde Coletiva, $21,2537-2546$

Gomes, M. A. V., Pinto, V. de O., \& Cassuce, F. C. da C. (2021). Determinantes da satisfação no atendimento das Unidades Básicas de Saúde (UBS). Ciência \& Saúde Coletiva, 26(4), 1311-1322.

Losco, L. N., \& Gemma, S. F. B. (2019). Sujeitos da saúde, agentes do território: o agente comunitário de saúde na Atenção Básica ao imigrante. Interface Comunicação, Saúde, Educação, 23.

Marinho, C. da S., \& Bispo Júnior, J. P. (2020). Supervisão de agentes comunitários de saúde na Estratégia Saúde da Família: entre controle, apoio e formação. Physis: Revista de Saúde Coletiva, 30(3).

Martins, J. R. T., Viegas, S. M. da F., Oliveira, V. C. de, \& Rennó, H. M. S. (2019). Vaccination in everyday life: experiences indicate Permanent Education. Escola Anna Nery, 23(4).

Massara, C. L., Murta, F. L. G., Enk, M. J., Araújo, A. D. de, Modena, C. M., \& Carvalho, O. dos S. (2016). Caracterização de materiais educativos impressos sobre esquistossomose, utilizados para educação em saúde em áreas endêmicas no Brasil. Epidemiologia E Serviços de Saúde, 25(3), 575-584.

Massaroli, M. V. (2016) Dinâmica de grupo no desenvolvimento de competências dos profissionais da saúde. Journal Health NPEPS, 1(2):278-286.

Oliveira, A. M. B., \& Medeiros, N. T. (2018). Fisioterapia na Residência Multiprofissional em Saúde da Família: relato de experiência. SANARE, 17(02):9199 .

Ramos, A. S. Aracaju em foco o modelo "Saúde Todo Dia": a Residência Multiprofissional em Saúde Coletiva em debate. In: Pinheiro, R., Mattos, R. A. (2006) Seminário do Projeto Integralidade, 6:65-70. Resumos Ampliados. CEPESC. ABRASCO.

Ribeiro, B. S., Carmo, E. A., Bonfim, E. S., Cardoso, T. S. G., Duarte, A. C. S., \& Boery, R. N. S. O. (2016). Metodologia da problematização no ensino em saúde: experiência com agentes comunitários de saúde. Rev. Enferm UFPE on line, 10(10):3907-3913.

Sanchez, M. P. C., Lemos, R. A., \& Veríssimo, M. L. R. (2017). Avaliação de materiais educativos para o cuidado e a promoção do desenvolvimento de crianças nascidas prematuras. Rev. Soc. Bras. Enferm. Ped., 17(2):76-82.

Simas, P. R. P., \& Pinto, I. C. de M. (2017). Trabalho em saúde: retrato dos agentes comunitários de saúde da região Nordeste do Brasil. Ciência \& Saúde Coletiva, 22(6), 1865-1876

Zerbeto, A. B., Carvalho, L., Rossa, T. A., \& Paula, D. (2020). Capacitação de agentes comunitários de saúde: integração entre universidade e atenção básica. Revista Brasileira de Extensão Universitária, 11(3):349-359. 